\title{
sciendo
}

\section{Effects of Ischemic Preconditioning as a Warm-Up on Leg Press and Bench Press Performance}

\author{
by \\ Luiz Guilherme da Silva Telles ${ }^{1,6}$, Luiz Cristiano Carelli2, Igor Dutra Bráz ${ }^{2}$, \\ Christian Junqueira², Estêvão Rios Monteiro1,3, Victor Machado Reis', \\ Jeferson Macedo Vianna ${ }^{5}$, Jefferson da Silva Novaes ${ }^{1,5}$
}

\begin{abstract}
Ischemic preconditioning (IPC) has been used to increase performance in sports. The aim of this study was to compare the acute effects of IPC with different warm-up methods on the number of repetitions and total volume in resistance exercise (RE). Sixteen healthy men recreationally trained in RE participated in this study. After the anthropometric evaluation and familiarization, a one-repetition maximum (1RM) test and retest were performed in the bench press $(B P)$ and in the leg press $45^{\circ}(L P)$ exercise. After these tests, participants were randomly assigned to one of the five protocols: a) IPC; b) SHAM; c) a specific warm-up (SW); d) aerobic exercise (AE), and e) active stretching $(A S)$ prior to performing 3 sets at 80\% 1RM until concentric failure. The number of repetitions was higher following IPC compared to the SW following three sets both for the BP and LP. Similarly, the number of repetitions for IPC was higher in comparison to SHAM following three sets for the LP. The number of repetitions was higher following IPC compared to AE following $1^{\text {st }}$ and $2^{\text {nd }}$ sets for the LP and following the $2^{\text {nd }}$ set for the BP. Finally, the number of repetitions was higher following IPC compared to AS following $1^{\text {st }}$ and $2^{\text {nd }}$ sets for the LP. The total volume was higher following IPC compared to SHAM, SW, AE, and AS for both the BP and LP. The IPC protocol increased the number of maximum repetitions and the total volume when compared to the other tested methods, thus indicating a better utilization during the pre-work warm-up. These results indicate positive associative responses to IPC with performance maintenance, which is of importance for both athletes and coaches.
\end{abstract}

Key words: ischemic preconditioning, resistance exercise, warm-up, performance, ischaemia.

\section{Introduction}

Ischemic preconditioning (IPC) is a method of blocking blood flow momentarily and then releasing it, causing blood reperfusion before performing physical exercise (Eltzschig and Eckler, 2011). In general, in addition to causing cardiovascular protective effects (Murry et al., 1986), IPC promotes muscular and organic improvements (Lawson and Downey, 1993; Pang et al., 1995). More recently, IPC has been used before sport tests (De Groot et al., 2010; Jean-StMichel et al., 2011; Foster et al., 2014; Kjeld et al., 2014; Paixão et al., 2014) or exercise (Marocolo et al., 2016a, 2016b; Paradis-Deschênes et al., 2016) aiming at increased performance.

The physiological mechanisms underpinning the effects of IPC on skeletal muscle are not fully understood (Lawson and Downey, 1993; Pang et al., 1995), but may be modulated by the release of nitric oxide (Kimura et al., 2007; Li

\footnotetext{
1 - Postgraduate Program in Physical Education, Federal University of Rio de Janeiro, Rio de Janeiro, Brazil.

2 - University Center of Volta Redonda, Volta Redonda, Rio de Janeiro, Brazil.

3 - Department of Physical Therapy, Augusto Motta University, Rio de Janeiro, Brazil.

4 - Research Center in Sports Sciences, Health Sciences \& Human Development, CIDESD, Vila Real, Portugal.

5 - Postgraduate Program in Physical Education, Federal University of Juiz de Fora, Minas Gerais, Brazil.

6 - Estácio de Sá University, Rio de Janeiro, Brazil.
} 
et al., 2012) and activation of the adenosine receptors (Liu et al., 1991; Schroeder et al., 1996), causing vasodilation after blood reperfusion (Kimura et al., 2007; Li et al., 2012) increasing the $\mathrm{O}_{2}$ extraction by the muscle (Tanaka et al., 2016; Paradis-Deschênes et al., 2016). In addition, the opening of adenosine triphosphate (ATP)dependent potassium $\left(\mathrm{K}^{+}\right)$channels is promoted by increasing the energy stocks after application of the method (Lawson and Downey, 1993; Pang et al., 1995).

De Groot et al. (2010) investigated IPC prior to the incremental cycling test, demonstrating an increase in $\mathrm{VO}_{2 \max }$. Since then, several studies have been performed applying IPC before running (Foster et al., 2014), swimming (Jean-St-Michel et al., 2011; Marocolo et al., 2015), diving and apnea (Kjeld et al., 2014), isometric exercise (Tanaka et al., 2016) and resistance exercise (RE) (Marocolo et al., 2016a, 2016b; Paradis-Deschênes et al., 2016, 2017). These studies verified the effects of IPC before RE performed in isolated and mono-articular groups. Thinking of greater practical applicability, our study investigated the effects of IPC as a form of a warm-up before multiarticular exercise for lower and upper limbs, most commonly used in a RE session. A warm-up is defined as the activity that precedes physical training to prepare the body's physiological, biomechanical and bioenergetic components (Garber et al., 2011). Different warmup methods, such as activity-specific (Fermino et al., 2005), stretching and aerobic exercise (Garber et al., 2011) have been used to improve muscle performance and joint amplitude.

Our hypothesis was that the effects generated by IPC before RE could improve muscle strength performance when compared to other warm-up protocols. In view of the above, the aim of the present study was to compare the effects of IPC with different warm-up methods before RE on force performance in leg and bench press exercises.

\section{Methods}

\section{Participants}

After explaining the risks and benefits of the research, participants signed an informed consent form elaborated according to the Declaration of Helsinki, which was approved by the local ethics committee (under protocol number 2114840). Sixteen men (age: $24.8 \pm 2.2$ years; body mass: $77.9 \pm 6.7 \mathrm{~kg}$; body height: 176.3 $\pm 5.3 \mathrm{~cm}$; body mass index: $25.1 \pm 2.2 \mathrm{~kg} / \mathrm{m}^{2}$; resistance training experience: $5.0 \pm 1.6$ years; test values of $1 \mathrm{RM}$ in the BP: $87.4 \pm 20.1 \mathrm{~kg}$ and in the LP: $284.4 \pm 61.8 \mathrm{~kg}$ ), normotensive, physically active in RE for at least one year volunteered for this study. Testing was performed using $G^{*}$ Power 3.1 software, following the recommendations of Beck (2013) to reduce the probability of type II error and to determine the minimum number of participants required for this investigation.

Participants who responded positively to at least one of the items of the Physical Activity Readiness Questionnaire / PAR-Q (Shephard, 1988), those who failed at least one of the testing sessions in the laboratory, who presented any type of osteomioarticular lesion in the upper or lower limbs and smokers, were excluded.

\section{Experimental Design}

Eight visits to the lab on non-consecutive days ( 3 to 7 days apart) were performed always at the same time of the day, to avoid the circadian influence. During the first visit anthropometric measurements and familiarization were carried out, and the questionnaires were applied. On the second and third visits, the 1RM test and retest were conducted. From the fourth to the eighth visit participants were randomly divided into five different groups with alternating and counterbalanced entry in the following experimental protocols: a) IPC; b) SHAM (false protocol); c) specific warm-up; d) aerobic exercise; and e) active stretching.

During the experiment, participants were instructed to refrain from exercise and to avoid caffeine, chocolate, nutritional supplements and alcohol intake for 48 hours before each test, sleep for a minimum of six hours the night before each testing session and not to perform the valsalva maneuver during the execution of the exercises. The experimental design of the study is presented in Figure 1.

\section{Anthropometrics}

Stature and body mass were measured using a stadiometer and a scale $(0-200 \mathrm{~cm})$ with $0.5 \mathrm{~cm}$ and $0.1 \mathrm{~kg}$ precision, respectively.

IPC and sham protocols

According to previous studies (Incognito et al., 2016; Marocolo et al., 2016a), IPC consisted of 4 cycles of unilateral 5-min occlusion (220 
$\mathrm{mmHg}$ ) followed by $5 \mathrm{~min}$ of reperfusion (0 $\mathrm{mmHg}$ ) in each thigh, using a $57 \times 9 \mathrm{~cm}$ pneumatic tourniquet (komprimeter Riester ${ }^{\circledR}$, Jungingen, Germany) applied around the subaxillary region of the arm. In this protocol, no warm-up was carried out before RE. Sham intervention was the same as in IPC, but only 20 $\mathrm{mmHg}$ was applied to the "occlusion phase". After the SHAM protocol, a specific warm-up was composed of two sets of 15 repetitions with $40 \%$ of $1 \mathrm{RM}$ for the bench press and leg press $45^{\circ}$ exercise. There was a $60 \mathrm{~s}$ rest interval between sets (Fermino et al., 2005). The total duration of both IPC and Sham was $40 \mathrm{~min}$. To make sure that participants would have restricted blood flow during the intervention, the absence of a pulse was verified according to previous studies (Loenneke et al., 2012).

The IPC and SHAM protocols are presented in Figure 2.

\section{Specific warm-up protocol}

The specific warm-up was composed of two sets of 15 repetitions with $40 \%$ of $1 \mathrm{RM}$ for the bench press and leg press $45^{\circ}$ exercise. There was a $60 \mathrm{~s}$ rest interval between sets (Fermino et al., 2005).

\section{Active stretching protocol}

The active stretching exercise in the upper limbs for the pectoralis major muscle was shoulder abduction with horizontal adduction. The exercise for the quadriceps, hamstrings and gluteus muscles was flexion and hip hyperextension with extended knees. For the application of the experimental protocols, all movements were brought to a position of slight discomfort (Garber et al., 2011). The dynamic active stretching method was applied with the volume of three sets of 30 repetitions, with a cadence of $1 / 0 / 1 / 0 \mathrm{~s}$ per $1 \mathrm{~min}$, attempting to reach greater range of movement in successive repetitions (Bacurau et al., 2009). There was a $30 \mathrm{~s}$ rest interval between cycles.

\section{Aerobic exercise protocol}

The aerobic exercise protocol consisted of walking on a treadmill at a light to moderate intensity between 30 and $60 \%$ of the HRR for 10 minutes (Garber et al., 2011).

$$
\left\{\mathrm{HRR}=\left[\left(\mathrm{HR}_{\max }-\mathrm{HR}_{\text {rest }}\right) \mathrm{x} \% \text { intensity }\right]+\mathrm{HR}\right\}
$$

To determine HR $\max$ the following formula was used: $\left[\mathrm{HR}_{\max }=208\right.$ - (age x 0.7)] (Tanaka et al., 2001).

1RM test

The training load prescription was evaluated through the 1RM test (ACSM, 2009). The evaluations were performed on the days of the visits to the laboratory as described in Figure 1. Exercises were performed bilaterally: the BP and LP (Buick@ Fitness Equipment, Rio de Janeiro, Brazil) using a 10-min recovery period between exercises. For the warm-up, each individual performed two sets of 5-10 repetitions at $40-60 \%$ with a 1-min rest interval between sets, considering the maximum perception of the individual's strength. After a 1-min rest interval, the third set was performed with 3 to 5 repetitions at $60-80 \%$ of the maximum perceived strength. After a rest period the force measurement was made, where up to 5 trials could be performed, adjusting the load before each new attempt. The recovery period between the trials was 3-5 min. The test was interrupted when the individual was unable to perform the movement correctly and the maximum load was considered in the repetition with the completed execution. In order to reduce the margin of error in data collection, the following strategies were adopted: (a) standardized instructions given before the tests so that each participant was aware of the procedures applied in data collection, (b) the participant was instructed on the proper technique of the exercise performance, (c) all participants received standardized verbal encouragement during testing, and (d) all tests were performed at the same time of day for each session. The highest load achieved between the two days was considered 1RM.

\section{RE session protocol}

The RE session was initiated shortly after the warm-up protocol and was composed of two upper and lower limb exercises, the BP and LP. Each exercise was performed in the volume of three sets with $80 \%$ of $1 \mathrm{RM}$ until concentric failure, with regular time cadence of 2/0/2/0 for all protocols, using a digital metronome (Soundbrenner, Berlin, Germany) (Wilk et al., 2018) with $90 \mathrm{~s}$ rest intervals between sets and two minute rest intervals between exercises.

\section{Rating of perceived exertion}

Before beginning the study, participants took part in a familiarization session with the 
OMNI-RES scale (Robertson et al., 2003). The RPE was assessed after the exercises of the upper and lower limbs.

\section{Statistical analysis}

Results are presented as mean \pm standard deviation. Normality was tested and not disregarded by the Shapiro-Wilk test and homoscedasticity was confirmed by the Levene's test. ANOVA with repeated measures was used to test interactions and to compare means. Possible significant differences were identified through the Bonferroni post-hoc test. In addition, the Wilcoxon's nonparametric test was used to compare the values of the subjective perception of effort under each condition. Effect size (ES) estimates were also calculated using the standardized mean difference to determine the magnitude of treatment effects. The magnitude of ES was interpreted using the scale proposed by Rhea (2004). All analyses were performed in SPSS software (SPSS Inc., V.21, Chicago, IL, USA) and the considered alpha value was 5\% $(p<0.05)$.

\section{Results}

A high intraclass correlation (ICC) was found for the 1RM test and retest for the BP $(0.977 ; 95 \%$ confidence interval $=0.923-0.993 ; p=$ $0.000)$ and the LP $(0.988 ; 95 \%$ confidence interval $=$ 0.966-0.996, $p=0.000$ ). The Shapiro-Wilk test did not find significant differences for the descriptive variables in the study group $(p>0.05)$, thus characterizing homogeneity.

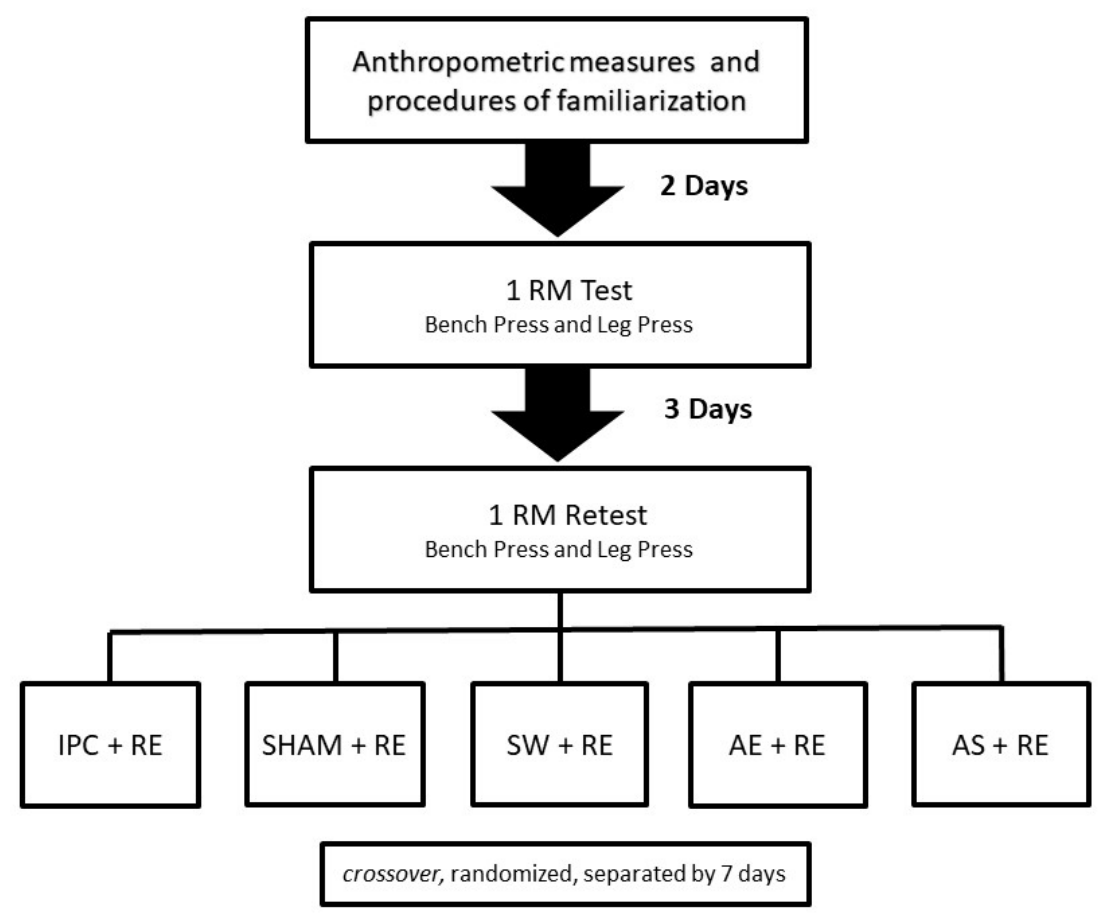

Figure 1

Experimental design of the study. 1 RM: one repetition maximum; IPC: ischemic preconditioning;

RE: resistance exercise; SW: specific warm-up; AE: aerobic exercise; AS: active stretching. 


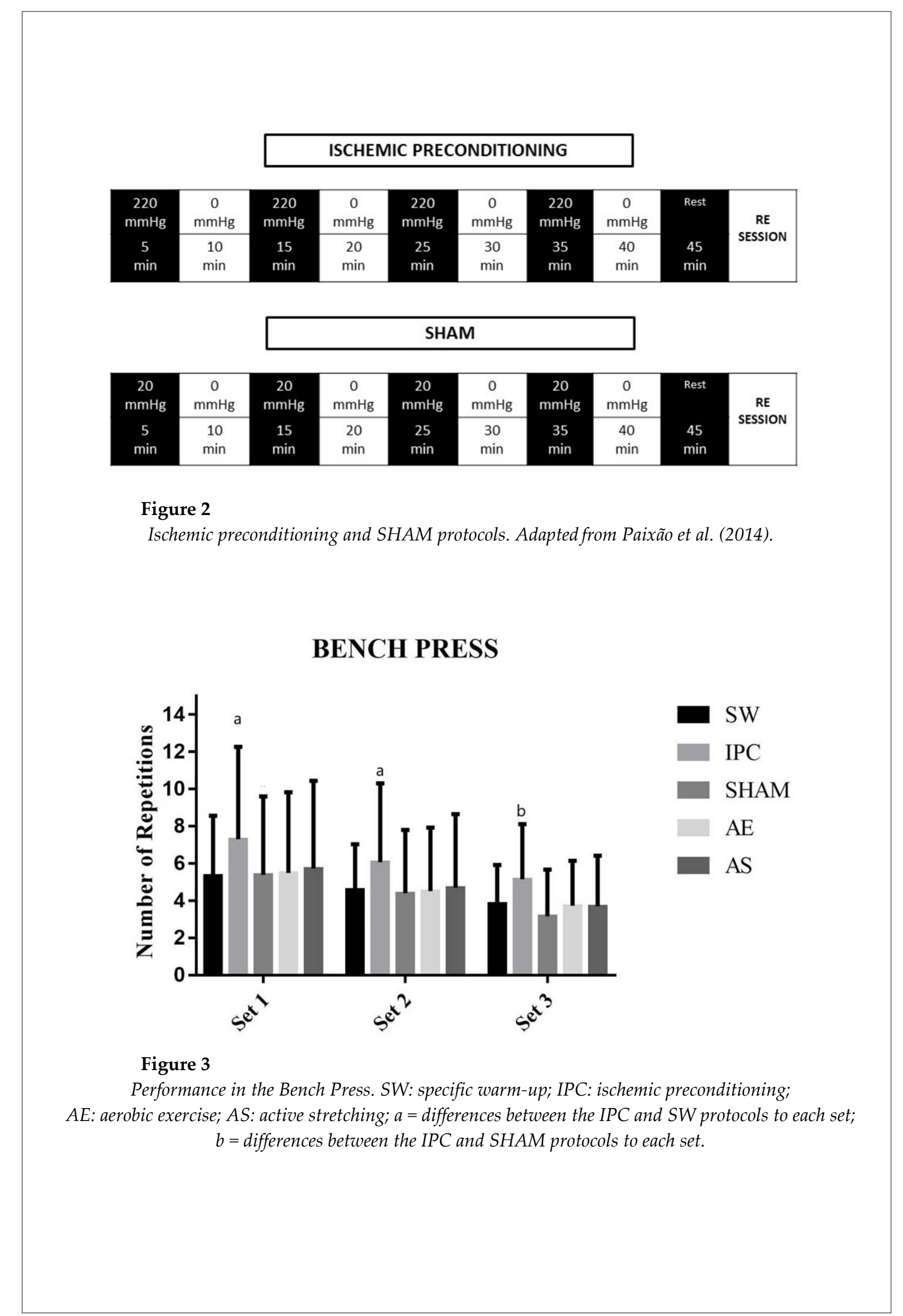

(c) Editorial Committee of Journal of Human Kinetics 


\section{LEG PRESS $45^{\circ}$}

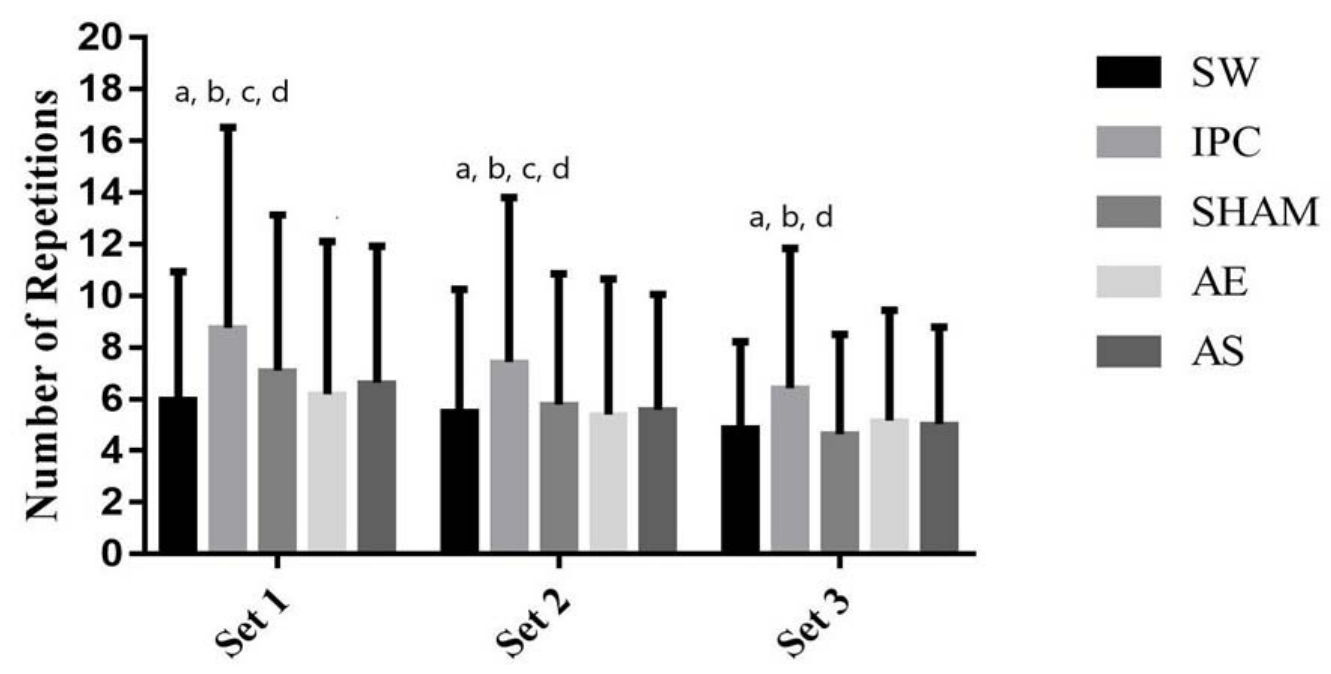

Figure 4

Performance in the Leg Press $45^{\circ}$. SW: specific warm-up; IPC: ischemic preconditioning;

AE: aerobic exercise; AS: active stretching; $a=$ differences between the IPC and SW protocols to each set; $b=$ differences between the IPC and SHAM protocols to each set; $c=$ differences between the IPC and AE protocols to each set; $d=$ differences between the IPC and AS protocols to each set.

\section{TOTAL VOLUME}

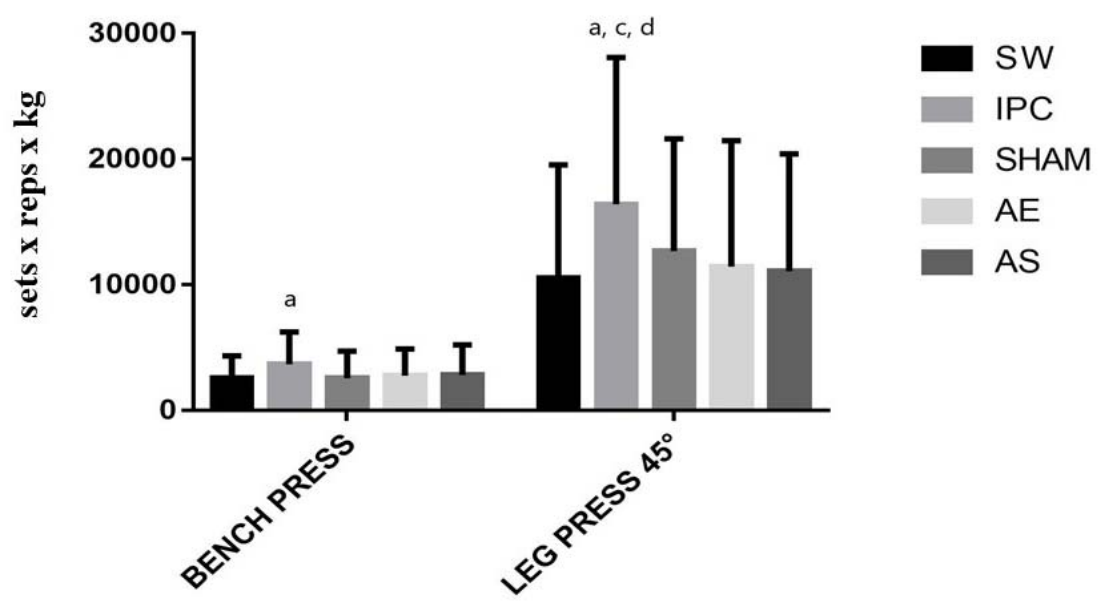

Figure 5

Total Volume in the two exercises. SW: specific warm-up; IPC: ischemic preconditioning; AE: aerobic exercise; AS: active stretching; $a=$ difference between IPC and SW protocols to each set; $c=$ differences between IPC and AE protocols to each set; $d=$ differences between IPC and AS. 


\section{RATING OF PERCEIVED EXERTION}

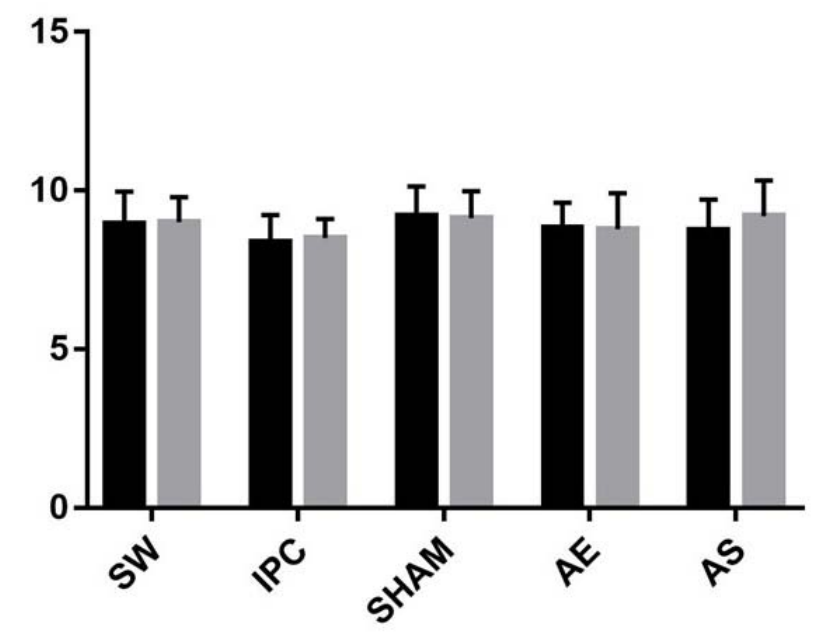

Bench Press

Leg Press $45^{\circ}$

Figure 6

Rating of Perceived Exertion in the two exercises. SW: specific warm-up; IPC: ischemic preconditioning; AE: aerobic exercise; AS: active stretching.

For the BP (Figure 3), the number of repetitions was higher following IPC compared to the SW in the first $(\Delta=41.8 \%, p=0.024)$ and the second set $(\Delta=43.6 \%, p=0.021)$. In the third set, the number of repetitions was higher following IPC compared to SHAM $(\Delta=46.8 \%, p=0.041)$.

For the LP (Figure 4), the number of repetitions was higher following IPC compared to the SW $(\Delta=50 \%, p<0.001)$, SHAM $(\Delta=25.3 \%, p=$ $0.039), \operatorname{AE}(\Delta=37.3 \%, p=0.001)$, and $\operatorname{AS}(\Delta=$ $37.3 \%, p=0.001)$ in the first set. In the second set, the number of repetitions was higher following IPC compared to SW $(\Delta=34.5 \%, p=0.004)$, SHAM $(\Delta=27.3 \%, p=0.026)$, AE $(\Delta=30.8 \%, p=0.010)$, and AS $(\Delta=36.4 \%, p=0.002)$. In the third set of the LP, the number of repetitions following IPC was significantly higher than following the SW $(\Delta$ $=41.4 \%, p=0.005)$, SHAM $(\Delta=39 \%, p=0.008)$, and AS $(\Delta=33.3 \%, p=0.027)$.

There were significant differences between IPC and the SW $(5481.1 \pm 1894,3823.8 \pm 1403.4, \mathrm{ES}=$
1.22, $p=0.012)$. In the comparison between the protocols in the LP over the total volume, significant differences were observed between IPC, SW, AE, and AS (24673.5 $\pm 8404.8,16904.2 \pm$ 4317.2, $\mathrm{ES}=1.86, p=0.004),(24673.5 \pm 8404.8$, $18518.2 \pm 4487.7, \mathrm{ES}=0.76, p=0.042),(24673.5 \pm$ 8404.8, $17673 \pm 4610.9, \mathrm{ES}=0.86, p=0.012$ ), respectively (Figure 5). As shown in Figure 6, the RPE scale was not significantly different between all protocols in both exercise modalities $(p>0.05)$.

\section{Discussion}

The purpose of the present study was to compare the effects of IPC with different warmup methods before RE on exercise performance. The main findings were that the IPC protocol increased the number of repetitions for the first and second sets of the BP exercise compared to the SW protocol and for the third sets compared to the SHAM protocol, and for all the sets of the LP exercise compared to all the other warm-up 
protocols, except for the third sets of the $\mathrm{AE}$ protocol. In addition, IPC showed increased total volume when compared to the SW protocol in the BP exercise and compared to the SW, SHAM and $\mathrm{AE}$ protocols in the LP exercise.

The comparison between BP protocols presented a significant difference between IPC and the SW in the 1st and 2nd sets, but no significant differences were observed between the other protocols. In the third sets, there was a significant difference between IPC and SHAM protocols, while no significant differences were observed between the other protocols. For the LP exercise, the analysis between protocols showed a significant difference between IPC and all the other protocols, although did not show differences between the other protocols in all sets. However, in the third set no differences between IPC and AE protocols were observed. In previous studies comparing different warm-up methods for $R E$, there were no significant differences between the traditional protocols, corroborating with the results of the present study (Fermino et al., 2005; Nicoli et al., 2007; Ribeiro et al., 2014). Interestingly, the IPC protocol was superior to traditional warm-up methods, demonstrating that IPC, in addition to promoting a muscle warm-up, increases strength performance.

Previous studies (Marocolo et al., 2016a, 2016b) investigating the effects of IPC on RE performance did not find significant differences when comparing IPC and SHAM, which does not corroborate the present study. Marocolo et al. (2016a) investigated the effects of IPC on the number of repetitions of the knee extension exercise over three maximal sets, while Marocolo et al. (2016b) evaluated the local and remote effects of IPC on performance over four sessions in Scott's elbow flexion exercise. In both studies, significant differences in the number of repetitions were identified when comparing both IPC and SHAM with the control protocol. However, no significant differences were found between IPC and the placebo.

Marocolo et al. (2016b) suggested that IPC should be considered as having a motivational effect, since in their previous research (Marocolo et al., 2016a) the SHAM protocol was able to generate an ergogenic effect in comparison to the control condition. On the other hand, ParadisDechênes et al. (2016) reported great improvement in strength $(\sim 12 \%)$ caused by IPC, when compared with SHAM, that could be attributed in part to competitive engagement of volunteers (psychological exertion), rather than being purely a result of neuromuscular function.

The great ergogenic effect $(\sim 33.7 \%)$ of IPC on the number of repetitions and total volume in RE observed in the present study agrees with previous studies (Paradis-Deschênes et al., 2016; Tanaka et al., 2016) showing benefits of IPC (12 to $17 \%)$ in dynamic and isometric force generation, respectively. The increase in force performance caused by IPC can be justified by physiological factors within the skeletal muscle (Incognito et al., 2016; Lawson and Downey, 1993; Pang et al., 1995; Salvador et al., 2016). In general, IPC is an intervention that causes positive changes in absorption kinetics of pulmonary O2 (Paganelli et al., 1989), in the systemic VO2 (De Groot et al., 2010), in the deoxygenation of muscular $\mathrm{Hb} / \mathrm{Mb}$ (Barbosa et al., 2015; Cleland et al., 2012; ParadisDeschênes et al., 2016; Tanaka et al., 2016) and in the opening of the ATP-dependent $\mathrm{K}+$ channels by increasing the energy stocks after IPC (Lawson and Downey, 1993), and in muscle vasodilation (Kimura et al., 2007; Li et al., 2012; Pang et al., 1995). In this sense, IPC can increase blood flow in the skeletal muscle (Incognito et al., 2016) and also improve exercise performance.

An increase of 2-4 repetitions in RE performance when IPC was applied herein, in comparison with the SHAM and other procedures, is in accordance with previous studies (Marocolo et al., 2016a, 2016b) which have shown mean improvements of 2 repetitions.

The RPE showed high values ( $\sim 9$ on a scale up to 10) under every condition of exercise, but did not present significant differences among them, which agrees with previous studies that evaluated the RPE in RE (Marocolo et al., 2016a, 2016b). Hence, it can be inferred that IPC does not influence the RPE in RE.

The present study had also some methodological limitations. The pneumatic tourniquet used in the study was manually handled during the alternation of cycles of ischemia-reperfusion, not allowing greater precision in the time of application. The volume between the warm-up protocols was not equalized, mainly due to the longer duration of IPC in comparison to the other warm-up 
protocols. However, all the protocols followed literature recommendations (Carvalho et al., 2012; Garber et al., 2011; McGowan et al., 2015).

The IPC protocol increased the number of maximum repetitions and the total volume when compared to the other tested methods, thus indicating a better utilization during the pre-work warm-up. These results indicate positive associative responses with performance maintenance. In addition, future research is warranted to assess the effects of different times of ischemia-reperfusion cycles in IPC, aiming to reduce the time of application of the method, increasing its practical applicability.

\section{Acknowledgements}

This work received funding from the Portuguese Foundation for Science and Technology, I.P. (project UID/DTP/04045/2019).

\section{References}

American College of Sports Medicine position stand. Progression models in resistance training for healthy adults. Medicine and Science in Sports and Exercise, 2009; 41: 687. Doi: 10.1249/MSS.0b013e318213fefb

Bacurau RFP, Monteiro GA, Ugrinowitsch C, Tricoli V, Cabral LF, Aoki MS. Acute Effect of a Ballistic and a Static Stretching Exercise Bout on Flexibility and Maximal Strength. J Strength Cond Res, 2009; 23: 304308. Doi: 10.1519/JSC.0b013e3181874d55

Barbosa TC, Machado AC, Braz ID, Fernandez IA, Vianna LC, Nobrega AC, Silva BM. Remote ischemic preconditioning delays fatigue development during handgrip exercise. Scand J Med Sci Sports, 2015; 25: 356-364. Doi: 10.1111/sms.12229

Beck TW. The importance of a priori sample size estimation in strength and conditioning research. J Strength Cond Research, 2013; 27: 2323-2337. Doi: 10.1519/JSC.0b013e318278eea0.

Carvalho FLP, Carvalho MCGA, Simão R, Gomes TM, Costa PB, Neto LB, Carvalho RLP, Dantas, EHM. Acute effects of a warm-up including active, passive, and dynamic stretching on vertical jump performance. J Strength Cond Research, 2012; 26: 2447-2452. Doi: 0.1519/JSC.0b013e31823f2b36

Cleland SM, Murias JM, Kowalchuk JM, Paterson DH. Effects of prior heavy-intensity exercise on oxygen uptake and muscle deoxygenation kinetics of a subsequent heavy-intensity cycling and kneeextension exercise. Appl Physiol Nutr Metab, 2012; 37: 138-148. Doi: 10.1139/h11-143

Cochrane DJ, Booker HR, Mundel T, Barnes MJ. Does intermittent pneumatic leg compression enhance muscle recovery after strenuous eccentric exercise? Int J Sports Med, 2013; 34: 969-974. Doi: 10.1055/s0033-1337944

De Groot PC, Thijssen DH, Sanchez M, Ellenkamp R, Hopman MT. Ischemic preconditioning improves maximal performance in humans. Eur J Appl Physiol, 2010; 108: 141-146. Doi: 10.1007/s00421-009-11952

Eltzschig HK, Eckle T. Ischemia and reperfusion-from mechanism to translation. Nature medicine, 2011; 17: 1391-401. Doi: $10.1038 / \mathrm{nm} .2507$

Fermino RC, Winiarski ZH, Da Rosa RJ, Lorenci LG, Buso S, Simão R. Influence of specific warm-up and stretching on muscle strength performance in 10 repetitions maximum. Rev Bras Ciê e Mov, 2005; 13: 25-32

Foster GP, Giri PC, Rogers DM, Larson SR, Anholm JD. Ischemic preconditioning improves oxygen saturation and attenuates hypoxic pulmonary vasoconstriction at high altitude. High Alt Med Biol, 2014; 15: 155-161. Doi: 10.1089/ham.2013.1137

Garber CE, Blissmer B, Deschenes MR, Franklin BA, Lamonte MJ, Lee IM, Nieman DC, Swain DP. American College of Sports Medicine Position Stand. The quantity and quality of exercise for developing and maintaining cardiorespiratory, musculoskeletal, and neuromotor fitness in apparently healthy adults: guidance for prescribing exercise. Med Sci Sports Exerc, 2011; 43: 1334-559. Doi: 10.1249/MSS.0b013e318213fefb 
Incognito AV, Burr JF, Millar PJ. The effects of ischemic preconditioning on human exercise performance. Sports Med, 2016; 46: 531-544. Doi: 10.1007/s40279-015-0433-5

Jean-St-Michel E, Manlhiot C, Li J, Tropak M, Michelsen MM, Schmidt MR, McCrindle BW, Wells GD, Redington AN. Remote preconditioning improves maximal performance in highly trained athletes. Med Sci Sports Exerc, 2011; 43: 1280-1286. Doi: 10.1249/MSS.0b013e318206845d

Kimura M, Ueda K, Goto C, Jitsuiki D, Nishioka K, Umemura T, Noma K, Yoshizumi M, Chayama K, Higashi Y. Repetition of ischemic preconditioning augments endothelium-dependent vasodilation in humans: role of endothelium-derived nitric oxide and endothelial progenitor cells. Arterioscler Thromb Vasc Biol, 2007; 27:1403-1410. Doi: 10.1161/ATVBAHA.107.143578

Kjeld T, Rasmussen MR, Jattu T, Bay H, Secher NH. Ischemic preconditioning of one forearm enhances static and dynamic apnea. Med Sci Sports Exerc, 2014; 46: 151-5. Doi: 10.1249/MSS.0b013e3182a4090a

Lawson CS, Downey JM. Preconditioning: state of the art myocardial protection. Cardiovasc Res, 1993; 27: 542-550. Doi: $10.1093 / \mathrm{cvr} / 27.4 .542$

Li XD, Cheng YT, Yang YJ, Meng XM, Zhao JL, Zhang HT, Wu YJ, You SJ, Wu YL. PKA-mediated eNOS phosphorylation in the protection of ischemic preconditioning against no-reflow. Microvasc Res, 2012; 84: 44-54. Doi: 10.1016/j.mvr.2012.04.002

Libonati JR, Cox M, Incanno N, Melville SK, Musante FC, Glassberg HL, Guazzi M. Brief periods of occlusion and reperfusion increase skeletal muscle force output in humans. Cardiologia, 1998; 43: 135560

Liu GS, Thornton J, Van Winkle DM, Stanley AW, Olsson RA, Downey JM. Protection against infarction afforded by preconditioning is mediated by A1 adenosine receptors in rabbit heart. Circulation, 1991; 84: 350-356. Doi: 10.1161/01.cir.84.1.350

Loenneke JP, Fahs CA, Rossow LM, Sherk VD, Thiebaud RS, Abe T, Bemben DA, Bemben MG. Effects of cuff width on arterial occlusion: Implications for blood flow restricted exercise. Eur J Appl Physiol, 2012; 112: 2903-2912.

Marocolo M, Da Mota GR, Pelegrini V, Appell Coriolano HJ. Are the Beneficial Effects of Ischemic Preconditioning on Performance Partly a Placebo Effect? Int J Sports Med, 2015; 36: 822-825. Doi: 10.1055/s-0035-1549857

Marocolo M, Willardson JM, Marocolo IC, Ribeiro Da Mota G, Simão R, Maior AS. Ischemic preconditioning and placebo intervention improves resistance exercise performance. J Strength Cond Res, 2016a; 30: 1462-1469. Doi: 10.1519/JSC.0000000000001232

Marocolo M, Marocolo IC, Da Mota GR, Simão R, Maior AS, Appell Coriolano HJ. Beneficial Effects of Ischemic Preconditioning in Resistance Exercise Fade Over Time. Int J Sports Med, 2016b; 37: 819-824. Doi: 10.1055/s-0042-109066.

McGowan CJ, Pyne DB, Thompson KG, Rattray B. Warm-up strategies for sport and exercise: mechanisms and applications. Sports Med, 2015; 45: 1523-1546. Doi: 10.1007/s40279-015-0376-x

Murry CE, Jennings RB, Reimer KA. Preconditioning with ischemia: a delay of lethal cell injury in ischemic myocardium. Circulation, 1996; 74: 112-436. Doi: 10.1161/01.cir.74.5.1124

Nicoli AIV, Cordova KO, Barreto ACLYG, Novaes JS. Influence of different warm-ups on the number of repetitions in resistance exercises. Arquivos em Movimento, 2007; 3: 42-55.

Paganelli W, Pendergast DR, Koness J, Cerretelli P. The effect of decreased muscle energy stores on the VO2 kinetics at the onset of exercise. Eur J Appl Physiol, 1989; 59: 321-326. Doi: 10.1007/bf02389805

Paixão RC, Da Mota GR, Marocolo M. Acute effect of ischemic preconditioning is detrimental to anaerobic performance in cyclists. Int J Sports Med, 2014; 35: 912-915. Doi: 10.1055/s-0034-1384588

Pang CY, Yang RZ. Zhong A, Xu N, Boyd B, Forrest CR. Acute ischaemic preconditioning protects against skeletal muscle infarction in the pig. Cardiovasc Res, 1995; 29: 782-788.

Paradis-Deschênes P, Joanisse DR, Billaut F. Ischemic preconditioning increases muscle perfusion, oxygen uptake, and force in strength-trained athletes. Appl Physiol Nutr Metab, 2016; 41: 938-944. Doi: 10.1139/apnm-2015-0561

Paradis-Deschênes P, Joanisse DR, Billaut F. Sex-Specific impact of ischemic preconditioning on tissue oxygenation and maximal concentric force. Front Physiol, 2017; 7: 674. Doi: 10.3389/fphys.2016.00674 
Rhea MR. Determining the magnitude of treatment effects in strength training research through the use of the effect size. J Strength Cond Res, 2004; 18: 918-920. Doi: 10.1519/14403.1

Ribeiro AS, Romanzini M, Schoenfeld BJ, Souza MF, Avelar A, Cirino ES. Effect of different warm-up procedures on the performance of resistance training exercises. Percept Mot Skills, 2014; 19: 133-145. Doi: $10.2466 / 25.29$

Robertson RJ, Goss FL, Rutkowski J, Lenz B, Dixon C, Timmer J, Andreacci J. Concurrent validation of the OMNI perceived exertion scale for resistance exercise. Med Sci Sports Exercise, 2003; 35: 333-341. Doi: 10.2466/25.29.PMS.119c17Zz

Salvador AF, De Aguiar RA, Lisboa FD, Pereira KL, Cruz RS, Caputo F. Ischemic preconditioning and exercise performance: a systematic review and meta-analysis. Int J Sports Physiol Perform, 2016; 11: 414. Doi: 10.1123/ijspp.2015-0204

Simão R, Farinatti PT, Polito MD, Maior AS, Fleck SJ. Influence of exercise order on the number of repetitions performed and perceived exertion during resistance exercises. J Strength Cond Res, 2005; 19: 152-156. Doi: 10.1519/1533-4287(2005)19<152:IOEOOT>2.0.CO;2

Shephard RJ. PAR-Q, Canadian Home Fitness Test and exercise screening alternatives. Sports Med, 1988; 5: 185-195.

Schroeder CA, Lee HT, Shah PM, Babu SC, Thompson CI, Belloni FL. Preconditioning with ischemia or adenosine protects skeletal muscle from ischemic tissue reperfusion injury. Journal of Surgical Research, 1996; 63: 29-34.

Tanaka D, Suga T, Tanaka T, Kido K, Honjo T, Fujita S, Hamaoka T, Isaka T. Ischemic Preconditioning Enhances Muscle Endurance during Sustained Isometric Exercise. Int J Sports Med, 2016; 37: 614-618. Doi: 10.1055/s-0035-1565141

Tanaka H, Monahan KD, Seals DR. Age-predicted maximal heart rate revisited. J Am Coll Cardiol, 2001;37: 153-6. Doi.org/10.1016/S0735-1097(00)01054-8

Wilk M, Goals A, Stastny P, Nawrocka M, Krzysztofik M, Zajac A. Does Tempo of Resistance Exercise Impact Training Volume? J Hum Kinet, 2018; 67: 241-250. Doi: 10.2478/hukin-2018-0034

\section{Corresponding author:}

\section{Luiz Guilherme da Silva Telles}

School of Physical Education and Sports, Federal University of Rio de Janeiro.

Rio de Janeiro, Brazil. Carlos Chagas Filho Avenue, 540 - University City, 21941-599.

E-mail: guilhermetelles@ufrj.br 